\title{
Robotic Total Mesorectal Excision using a Wristed Suction- irrigation Device for Efficient Traction and Visualization
}

\author{
Sung Uk Bae, M.D., Woon Kyung Jeong, M.D., Seong Kyu Baek, M.D. \\ Department of Surgery, School of Medicine, Dongsan Medical Center, Keimyung University, Daegu, Korea
}

In rectal cancer surgery, gentle opening of the plane by continuous traction and optimized visualization is essential. Recently, a wristed robotic suction-irrigation device was developed for efficient traction of the rectum and good surgical visualization. This video shows a technique of robotic total mesorectal excision using a wristed robotic suction-irrigation device. A 74-year-old woman with rectal cancer had a biopsy-proven adenocarcinoma within $9 \mathrm{~cm}$ of the anal verge. She underwent totally robotic total mesorectal excision using a dual-docking technique. Total procedure time was 445 minutes. The patient was discharged on postoperative day 8 without any complications. Total number of lymph nodes harvested was 12, and proximal and distal resection margins were 11.2 and $4.7 \mathrm{~cm}$, respectively. Totally robotic total mesorectal excision using a wristed robotic suctionirrigation device was an efficient and useful procedure for rectal cancer.

Keywords: Robotic surgical procedures, Laparoscopy, Rectal neoplasms, Lymph node excision
Received July 10, 2017

Accepted August 2, 2017

Corresponding author

Seong Kyu Baek

Department of Surgery, School of

Medicine, Dongsan Medical Center,

Keimyung University, 194 Dongsandong, Jung-gu, Daegu 41931, Korea Tel: $+82-53-250-8050$

Fax: +82-53-250-7322

E-mail:sgbeak@dsmc.or.kr

Supplementary video file: This article contains supplementary material (https://doi.org/10.7602/ jmis.2017.20.3.120).

This is an Open Access article distributed under the terms of the Creative Commons Attribution Non-Commercial License (http:// creativecommons.org/licenses/by-nc/4.0/) which permits unrestricted non-commercial use, distribution, and reproduction in any medium, provided the original work is properly cited.

\author{
Copyright () 2017 The Journal of Minimally \\ Invasive Surgery. All rights reserved.
}

\section{INTRODUCTION}

In rectal cancer surgery, gentle opening of the plane by continuous traction and optimized visualization is essential. In robotic pelvic dissection, an excellent stereoscopic view can be obtained with high illumination, a stable camera platform, and filtering of physiologic tremors. ${ }^{1-4}$ Recently, a wristed robotic suction-irrigation device was introduced for efficient traction and good visualization in rectal cancer surgery. This video shows a technique of robotic total mesorectal excision using a wristed robotic suction-irrigation device.

\section{PROCEDURE}

A 73-year-old woman with rectal cancer had a biopsyproven adenocarcinoma within $9 \mathrm{~cm}$ of the anal verge. She underwent totally robotic total mesorectal excision using a dual-docking technique. During pelvic dissection, the console surgeon controlled a $45^{\circ}$, articulating, 8-mm robotic suctionirrigation device, which was used not only for suction and irrigation but also for efficient traction of the rectum to various directions via the R2 arm (the surgeon's left hand). Total procedure time was 445 minutes. The patient was discharged on postoperative day 8 without any complications. Pathologic examination of the specimen revealed a T3N2aM0 adenocarcinoma without circumferential margin involvement. Total 
number of lymph nodes harvested was 12, and proximal and distal resection margins were 11.2 and $4.7 \mathrm{~cm}$, respectively.

\section{DISCUSSION}

Gentle opening of the plane by continuous traction and optimized visualization in rectal cancer surgery is essential. A wristed robotic suction-irrigation device offers surgeons precise control of a fully articulated suction-irrigation instrument, excellent autonomy at the control console, access to difficultto-reach anatomy, easy maintenance of a clear surgical field, optimized visualization, and efficient traction of the rectum. In this case, the magnified robotic view using a wristed robotic suction-irrigation device enabled determination of the correct surgical plane and $45^{\circ}$ articulating snake wrist with blunt tip provided effective traction of the rectum. Totally robotic total mesorectal excision using wristed robotic suctionirrigation device for rectal cancer was an efficient and useful procedure.

\section{REFERENCES}

1) Bae SU, Min BS, Kim NK. Robotic Low Ligation of the Inferior Mesenteric Artery for Rectal Cancer Using the Firefly Technique. Yonsei Med J 2015;56:1028-1035.

2) Lim DR, Bae SU, Hur H, et al. Long-term oncological outcomes of robotic versus laparoscopic total mesorectal excision of midlow rectal cancer following neoadjuvant chemoradiation therapy. Surg Endosc 2017;31:1728-1737.

3) Bae SU, Saklani AP, Hur H, et al. Robotic and laparoscopic pelvic lymph node dissection for rectal cancer: short-term outcomes of 21 consecutive series. Ann Surg Treat Res 2014;86:76-82.

4) Kim NK, Kim MS, Al-Asari SF. Update and debate issues in surgical treatment of middle and low rectal cancer. J Korean Soc Coloproctol 2012;28:230-240. 